\title{
EAl Endorsed Transactions

\section{SmartPPM: An Internet of Things Based Smart Helmet Design for Potholes and Air Pollution Monitoring}

\author{
Vibhutesh Kumar Singh ${ }^{1}$, Himanshu Chandna ${ }^{1}$, Nidhi Upadhyay ${ }^{2, *}$ \\ ${ }^{1}$ Indraprastha Institute of Information Technology (IIIT-Delhi), New Delhi, India \\ ${ }^{2}$ Circuit Uncle ${ }^{\mathrm{TM}}$, Uttam Nagar, New Delhi, India
}

\section{Abstract}

In a country with an extensive road network, it is very tough for authorities to identify and repair the potholes on time, which emerge due to casual wear and tear of the road. These potholes are dangerous for unsuspecting high-speed vehicles and results in multiple life-threatening accidents year-round. Apart from potholes, another severe concern about the time spent on roads is air pollution. Breathing the polluted air, mainly containing the particulate matter that has a diameter of fewer than 2.5 micrometers, is toxic to humans. In this work, we have judiciously designed an Internet of Things based smart helmet, which uses crowdsourcing to report potholes and collect crucial on-road air pollution data so that a person could avoid risk to life and health. We have also introduced the novel concept of remembrance factor and severity index, which could be useful in dealing with the stale and invalid pothole data in the database.

Received on 02 March 2019; accepted on 17 March 2019; published on 26 April 2019

Keywords: smart helmet, potholes, pollution, monitoring, iot, internet of things, sensors, smart devices, demonstration, prototype

Copyright (c) 2019 Vibhutesh Kumar Singh et al., licensed to EAI. This is an open access article distributed under the terms of the Creative Commons Attribution license (http://creativecommons.org/licenses/by/3.0/), which permits unlimited use, distribution and reproduction in any medium so long as the original work is properly cited.

doi:10.4108/eai.13-7-2018.163833

\section{Introduction}

Vast countries (e.g., USA, India), rely heavily on their road infrastructure for passenger and goods transport. In particular, India has a network of over 5.89 million kilometers (equivalent to 3.66 million miles) of roads as on 31st March 2017 (second largest in the world), as reported in Basic Road Statistics of India (2016-17) [1]. Since then, India has seen its road network growth at a phenomenal pace (about an average of 27 kilometers of highways have been constructed every day) [2]. While overspeeding and drunk driving is the primary causes of road casualties and damage to vehicles, these get further intensified by ill-maintained roads [3]. Potholes, in particular, are dangerous when the vehicle driver is unaware of its existence, and at high speed, they can cause serious accidents. With a considerable road

\footnotetext{
^ The code, data and recorded interviews associated with this project is available at: https://github.com/circuituncle/SmartPPM.

*Corresponding author. Email: admin@circuituncle.com
}

network and ever-increasing vehicular traffic, in a country like India, road authorities find it very difficult to locate every pothole on time, which often becomes the cause of a road accident.

The media reports and surveys often support the claim that potholes are deadly, e.g., a 2018 study revealed that in the year 2017, potholes claimed 3597 lives across India, which is equivalent to losing ten precious lives per day [4]. [4] also suggested that the reported fatalities due to potholes were $50 \%$ higher than the year 2016. Although potholes seem to be a developing nation's problem, instead, it's a global phenomenon. Globally a large amount of money is spent to locate and fill these potholes, which can occur randomly throughout the year [5] [6]. The randomness of pothole's occurrence is the primary difficulty in tracking them.

Several years back, pothole detection was mostly a manual task, which was usually labor-intensive and time-consuming. But in the current times, various ways exist to automate the detection process [7], which 
utilizes techniques like (1) manual notification using mobile applications, (2) computer vision, and (3) sensor data feedback. In a manual mobile application based notification system, the vehicle rider using a mobile application (e.g., Sukhad Yatra mobile application for India) has to take a photograph or provide textbased feedback of the potholes, which is then notified to the concerned authorities. This method is rather impractical since the vehicle rider can't just stop to take a photograph or provide a text-feedback of the pothole, amidst nearby vehicles at high speed. Also, these systems offer one-way feedback, which could not be further used to alert other vehicle riders about pothole's location. Due to this, an automated approach for pothole detection is a reasonable choice.

For an automated pothole detection, various works exists in the literature, e.g., [8], [9], [10], which suggests the use of computer vision $(\mathrm{CV})$. The $\mathrm{CV}$ based approach is further enhanced in terms of accuracy by combining it with machine learning (ML) techniques, as suggested in works like [11] - [14]. These techniques are applied either on thermal or visual imaging data, and they require a dedicated imaging device, i.e., a camera which mounted on the vehicle. This technique loses its appeal in low to medium income countries, where the vehicle riders are more concerned about the device's theft [15] than personal safety. Considering the practicality of ML and CV based pothole detection schemes, they are often trained on limited sampled datasets, e.g., < 200 in [11]; thus, the reported high level of accuracy of their trained classifiers may not be achieved in a real-world situation. Also, these methods may fail to detect potholes if the imaging environment becomes unfavorable, e.g., a detection system trained on images of daytime, may not work in the night and on the waterlogged roads. These $\mathrm{ML}$ and $\mathrm{CV}$ based methods are also susceptible to training bias. If the training is done using an imbalanced dataset [16], e.g., a classifier trained only on European roads may not work in other countries as the pothole, soil and road texture may differ. Since most CV and ML-based pothole detection systems concern only about the realtime detection, and doesn't let its user know pothole's location a priori; which makes this approach impractical for the use with high-speed road traffic.

Research including [17], [18] and [19] suggests the use of sensor data (particularly accelerometer), for pothole detection. Once the vehicle encounters a pothole, the accelerometer output shows a sudden spike, which is then combined with the instantaneous global positioning satellite (GPS) location to register a pothole. But the sensor-based approach of automatic pothole detection preassumes that the vehicle always passes through the pothole, which is contrary to any real-world scenario, where every vehicle driver tries his best to avoids potholes. Works including [17] - [19] also fails to provide any solution for dealing with the stale pothole data which gets collected up in the system once authorities start filling these potholes.

Apart from potholes, another concerning issue about the time spent on-road is the widespread air pollution. In a developing country, air quality and pollution, despite being one of the sustainable development goals set by the United Nations Organization [20], it is mostly neglected. Harmful emissions from vehicle exhausts contribute significantly to respiratory diseases [21]. Although multiple air pollution monitors have been set up to report the air quality index (AQI) in many parts of the world, the AQI data cannot be utilized by an individual road traveler to decide the route, which avoids the area of high air pollution level. The techniques used by navigation applications also do not take into account the quality of roads and air pollution.

Based on these needs, we propose SmartPPM: automated crowdsourcing based Smart helmet design for efficient Potholes and Pollution Monitoring. We choose two-wheeler drivers/riders as device carriers (the smart helmet), who are responsible for the collection of data. As the law around the world requires a person to wear a helmet while traveling on a two-wheeler, hence a smart helmet would be the best solution for the said problem. The data collected by the smart helmet is sent wirelessly to a mobile application installed on the rider's smartphone. The mobile application communicates with the cloud server to push any positive detection of a pothole and continuous pollution data in a database. The crowdsourced data then used by the mobile application to inform (through phone vibrations) the users in realtime to slow down when approaching a pothole or could be used by a navigation service provider to choose a route that is relatively pothole and pollution-free. This apriori pothole notification feature can prove to be very useful in alerting vehicle riders if the roads are waterlogged. Along with these features, we also propose a novel remembrance factor and severity index to deal with the problem of stale data. For this work, we incorporate a human-centric design approach, which includes feedback from real bike riders.

\section{Design details}

\subsection{A human centric design approach}

We have interviewed 11 individuals in New Delhi, India who use every day a two-wheeler to commute. The individuals were approached for the interview at random in terms of their age, sex, job profiles, etc. Some conclusions drawn from these interviews are listed.

- An individual would prefer the shortest route, even if the road has multiple potholes. 
- Two-Wheeler riders prefer to use a familiar route rather than the unknown. In a usual course, the pothole's locations are in the rider's memory, and thus avoiding a pothole is possible.

- If a priori information about the pollution and potholes is available to individuals, it will affect their decision to take up a route. Particularly they are keen to take a route having less pothole and pollution.

- Most of them don't have any knowledge of existing methods to report the potholes.

- For the pothole feedback mechanism, interviewed individuals have shown a preference to methods which doesn't involve any hand or leg movement, as this could dis-balance them while riding a bike.

- A feedback mechanism should be present to indicate riders about the approaching potholes from a safer distance so that they would be able to slow down on time.

- Most individuals are willing to contribute if they think their actions will help others.

- Although obligatory by the law, two-wheeler riders think that these innovations will make helmets more attractive to people and make the journey safer.

Based on these interviews, we have decided on a head nodding gesture (3-Times nodding) based feedback for a positive pothole report so that hand/legs need not be moved, whereas the pollution data is automatically collected at specified intervals. Along with the local daily bike commuters, the package/food delivery service providers could also be approached for being a possible contributor.

\subsection{System Architecture}

Fig. 1 shows our SmartPPM's system architecture. As the helmet has to be powered up with a rechargeable battery, we have taken care to make the smart helmet with the least number of components so that it can operate for a long time, and thus we have re-used several smartphone features, e.g., internet, and GPS access.

Whenever a rider with a SmartPPM helmet makes a positive gesture suggesting the presence of a pothole, the helmet sends an acknowledgment packet to the mobile application through a Wi-Fi link, which then coupled by the current GPS location pushed to a cloud based database as JavaScript object notation (JSON) packet by the mobile application whenever the internet access is available otherwise buffered. Meanwhile, the helmet concurrently at intervals of 1 seconds transmits the air pollution data to the mobile application ${ }^{1}$, which is coupled with GPS location data and the information is buffered in the phone memory for 10 minutes before pushing into the database at the cloud server. This data flow from the smart helmet to the SmartPPM cloud could be visualized in Fig. 2 that represents the data flow diagram of the implemented system.

When an user equipped with the mobile application approach towards the pothole, the user is notified through rhythmic phone vibrations if within the $150 \mathrm{~m}$ range. Apart from the alert mechanism, access to the SmartPPM's pollution and pothole database could be granted to map based routing applications e.g., Google Maps, which when combined with their existing routing algorithms could provide users a relatively pothole and pollution free route. Fig. 3 is the use case diagram of an user's interaction with the SmartPPM system that shows the relationship between the user and the different use cases in which the user is involved.

\subsection{Components Used and Specifications in Smart Helmet}

To build our working prototype, we have utilized various components which are listed out in this section. The parts are selected, keeping in mind costeffectiveness, compactness, current consumption, and developer friendliness so that it could be put into production quickly.

- Programmable WiFi Module (ESP8266 ESP-12S) [22]: This module enables a low powered Wi-Fi communication link and could be programmed to transmit pothole and pollution data between the helmet and mobile application or to push JSON packets containing pothole and pollution data directly to the cloud server subject to the availability of the internet. This module can be interfaced with various sensors, and capable of processing data eradicating the need of an additional processing unit.

For our prototype ESP8266 ESP-12S module is operated in modem sleep mode, in which the WiFi modem is switched off when any transmission isn't happening. In [23] authors assess the current consumption be $27.0 \mathrm{~mA}$, for transmissions at every 1 second in modem sleep mode. The battery connected to power up this module could be charged up directly by connecting a universal serial bus (USB) cable,

\footnotetext{
$\overline{{ }^{1} \text { The choice of } 1 \text { second transmit interval was based on [23]'s }}$ extensive power analysis for the ESP8266 module, which helped us to draw a theoretical power budget while planning the components of the prototype. One can choose a longer/shorter transmit interval depending on the device's power usage constraints, or if wanted to collect more pollution data samples per unit time.
} 


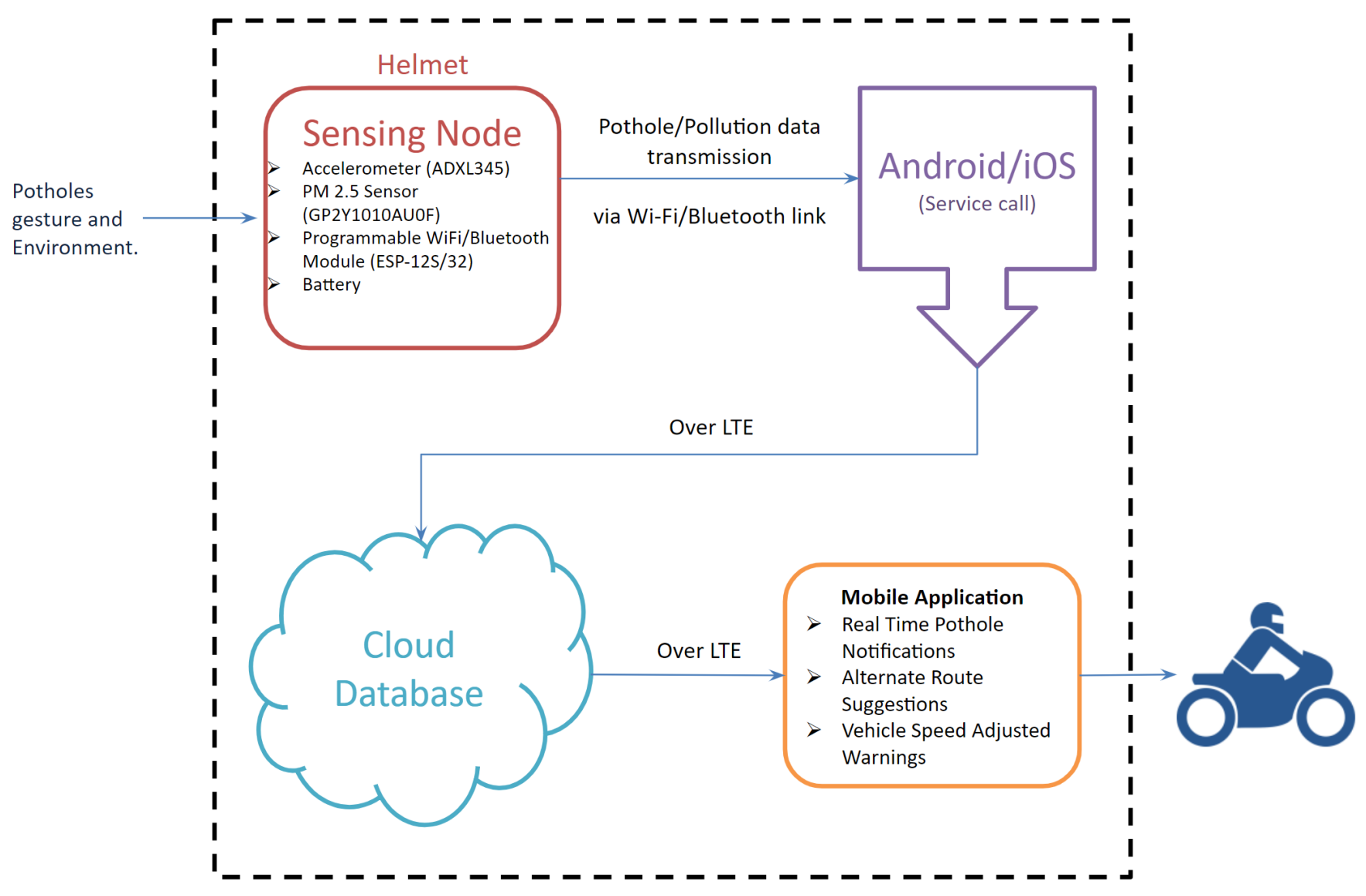

Figure 1. System architecture of SmartPPM system.

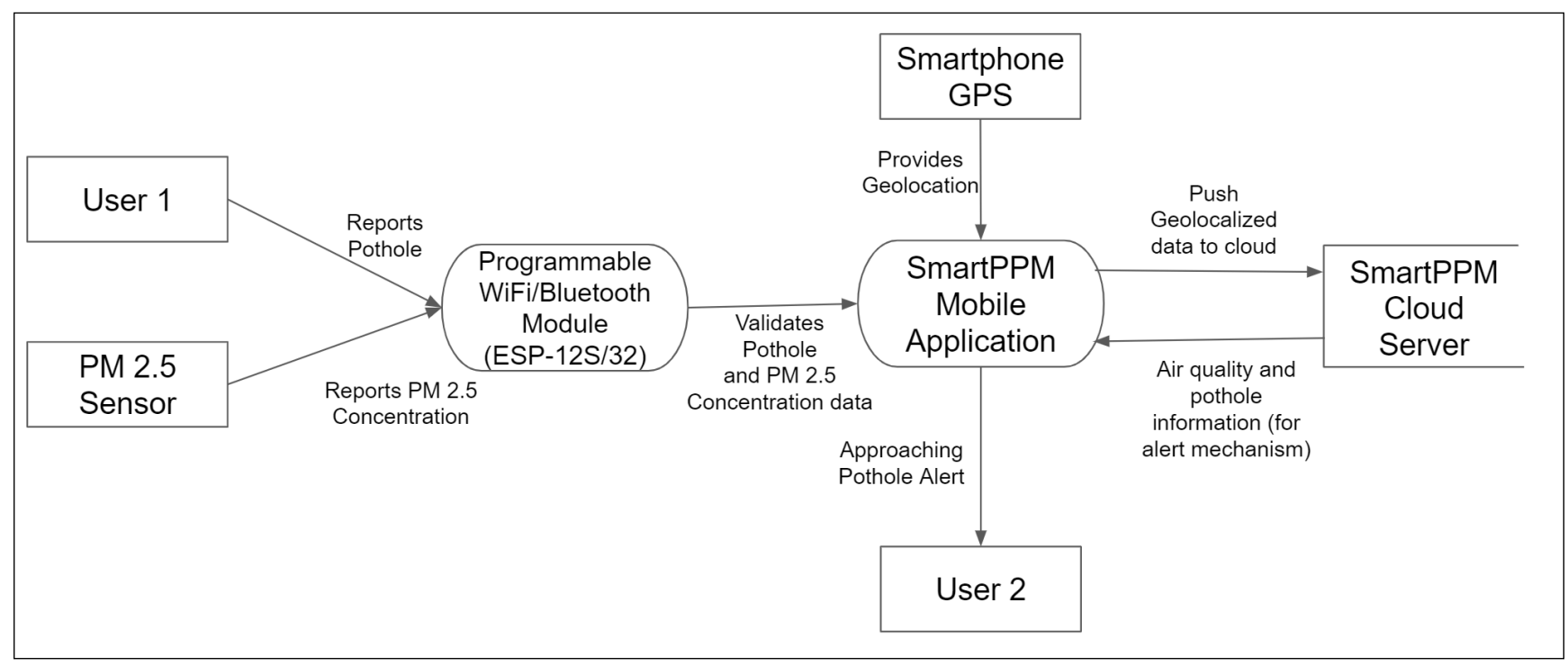

Figure 2. Data Flow diagram for SmartPPM system. Here User 1 is the person wearing the helmet whereas User 2 is the person using the mobile application to receive the approaching pothole alert. User 1 and 2 could be the same person, if wearing the SmartPPM helmet and utilize the mobile application to receive approaching pothole alerts.

thus eradicating the need for battery removal from the helmet at every recharge. Another suitable low power alternative of ESP-12S is ESP32 module [24], which has inbuilt Bluetooth and 


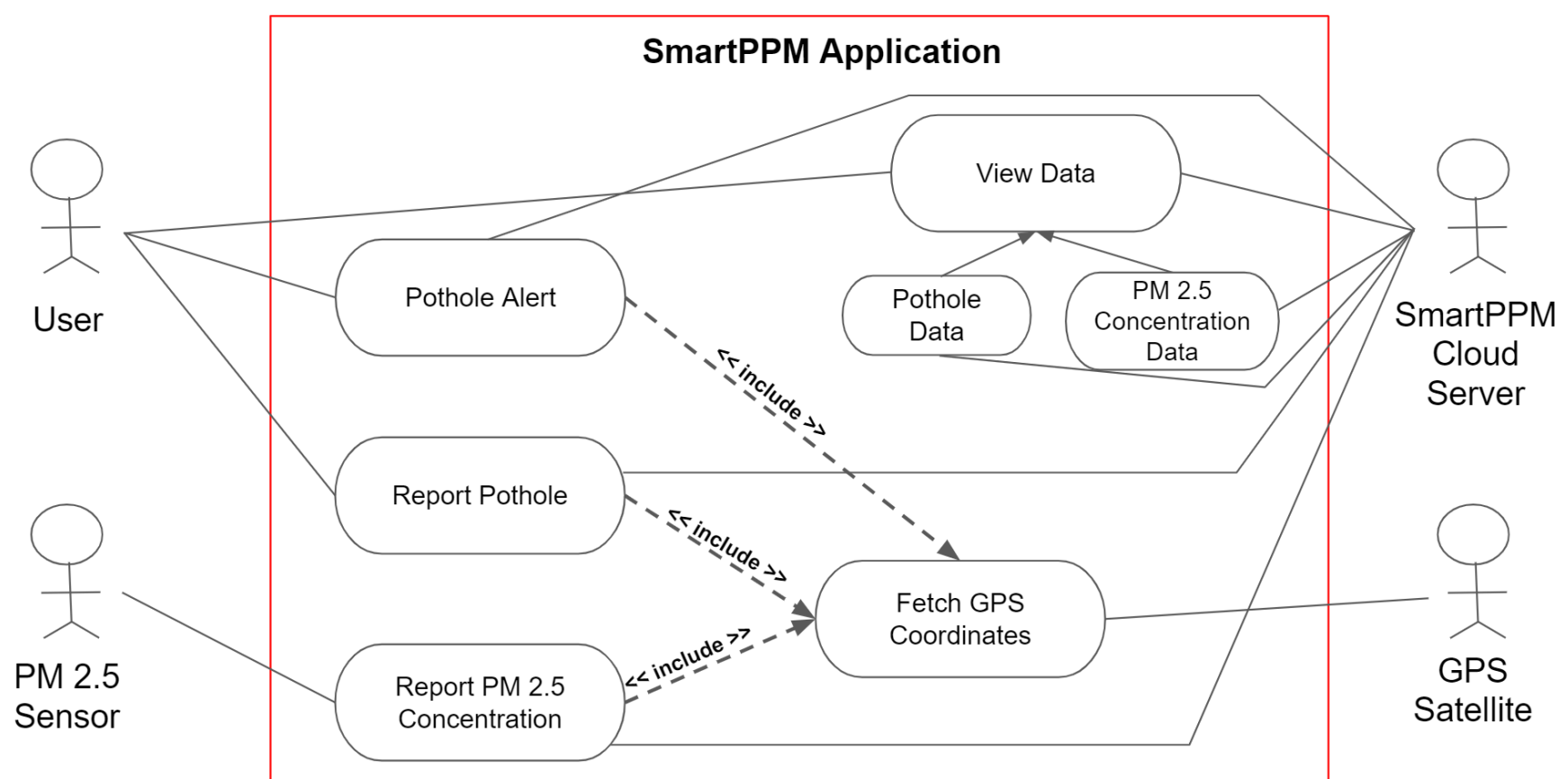

Figure 3. Use case diagram of SmartPPM system.

WiFi communication capabilities, with similar processing capabilities and hardware form factor as ESP-12S.

- Accelerometer (ADXL345) [25]: The accelerometer is utilized to recognize a pothole gesture (three times nodding) performed by the two-wheeler rider. As the pothole can occur at any time, the accelerometer is always in measurement mode and have a current consumption of $40 \mu \mathrm{A}$. The accelerometer is used to gather 3-axis acceleration data at a fixed resolution of 10-bits.

- PM 2.5 Sensor (GP2Y1010AU0F) [26]: This sensor measures the PM 2.5 concentration in the air, with a sensitivity of $0.5 \mathrm{~V}$ per $0.1 \mathrm{mg} / \mathrm{m}^{3}$. Since there is a linear relation of the output voltage and PM 2.5 concentration, the pollution estimation in terms of the Air Quality Index (AQI) could be quickly done using US EPA AIR-NOW concentration to AQI conversion scale. This module has a typical current consumption of $11 \mathrm{~mA}$.

- Battery (a Lithium Polymer battery) : We have used a 1200 mAh 3.7 V LiPo battery satisfying the voltage requirement of all the components used, and is theoretically sufficient for about 32 hours of continuous operation of the smart helmet. On actual testing, we found it to be adequate for 24 hours of operation, which is sufficient for a 3 day, 8 hours daily operation.

\subsection{Implementation Specifics}

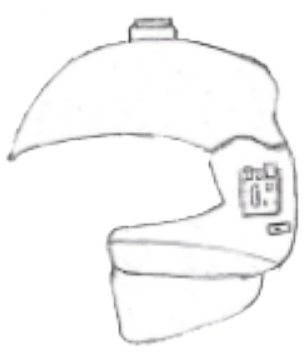

Left View

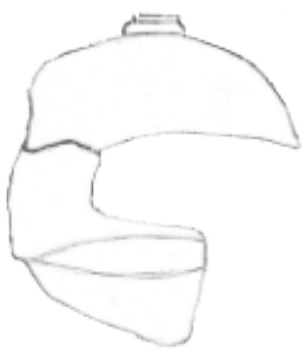

Right View

PM 2.5 Sensor (GP2Y1010AU0F)

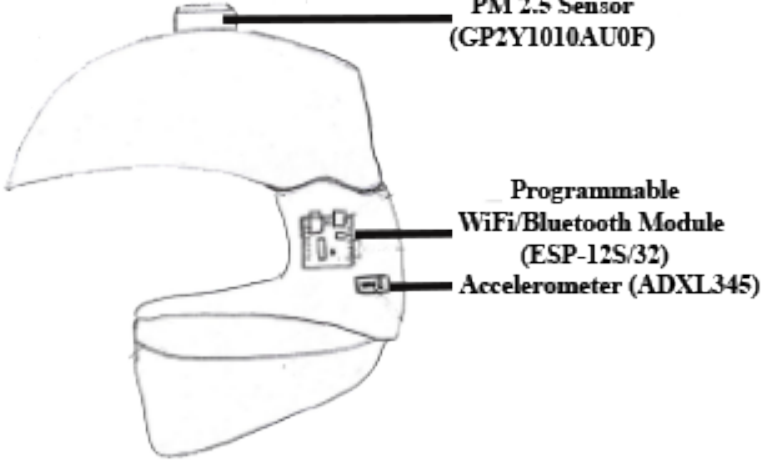

Figure 4. SmartPPM helmet schematic, showing the fitting scheme of the sensors on and inside the helmet.

Fig. 4, shows schematic of the helmet and the fitting location of the sensors inside and over it. We have mounted the PM 2.5 sensor on the top of the helmet. 
The ESP-12S/32, along with the accelerometer and the battery is located near the left ear side area of the helmet. All the components are internally wired to complete the circuit. The ear side foam cutouts to fit the components are shown in Fig. 5. Fig. 6.(a) refers to our

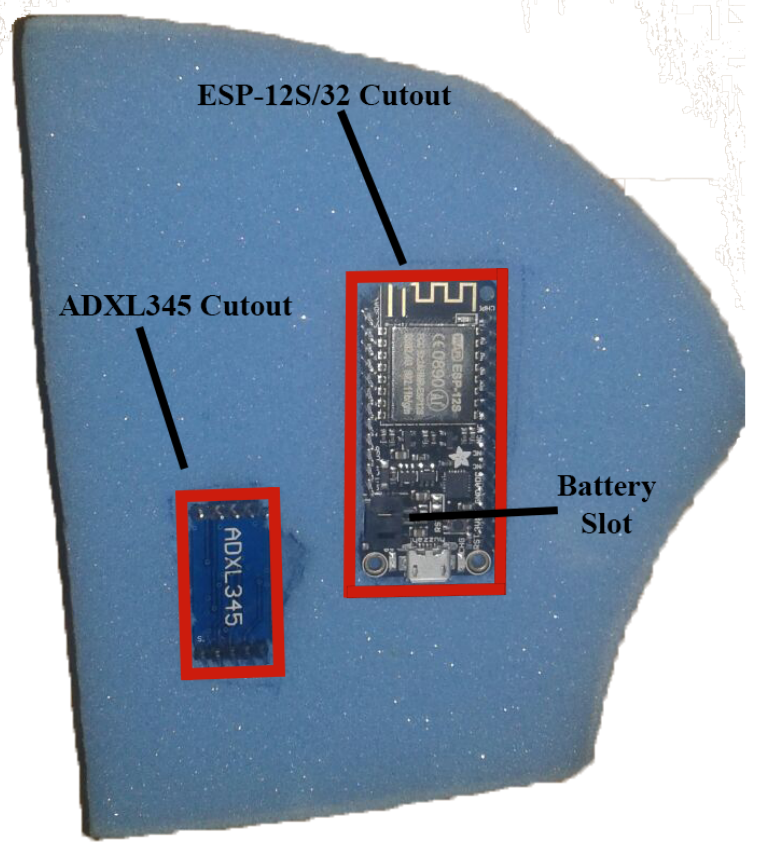

Figure 5. Accelerometer (ADXL345) and Programmable $\mathrm{WiFi}$ /Bluetooth Module (ESP-12S/32) cutout for internal fitting inside the helmet.

SmartPPM mobile application default screen showing the number of potholes detected by the system per month as a bar graph; this information is also visualized on the map above it. The user can directly switch to pollution data from this screen with the button provided. Fig. 6.(b), refers to the SmartPPM mobile application screen showing a line graph for monthly average PM 2.5 concentration encountered by the user, while on the road. The $y$-axis of the graph shows the AQI calculated through the scale provided by US EPA AIR-NOW and we set $250 \mathrm{AQI}$ as a danger to health level. The data shown is the result of our test runs on New Delhi (India) roads.

Fig. 7, shows the implemented helmet with mounted PM 2.5 sensor (GP2Y1010AU0F) on the top, alongwith the accelerometer, battery and programmable $\mathrm{Wi}$ Fi module on the left inner side of the helmet.

\subsection{Pothole remembrance factor and severity index}

Many current works, including [10], [17], fail to acknowledge the fact that authorities over time fill up the potholes and not able to automatically update/delete the pothole data when it is no longer present at the previously detected site. The only (a)
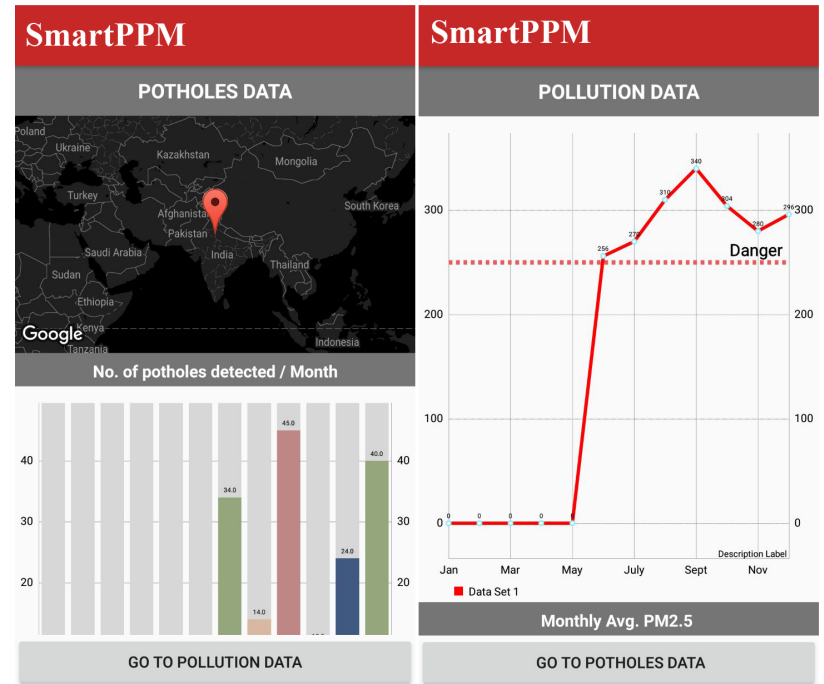

Figure 6. (a). SmartPPM mobile application screen displaying total pothole data collected during our trial. The number of pothole detected per month is displayed through bar graph as well as through the map, and (b)The graph depicting monthly average PM 2.5 encountered by a particular user.

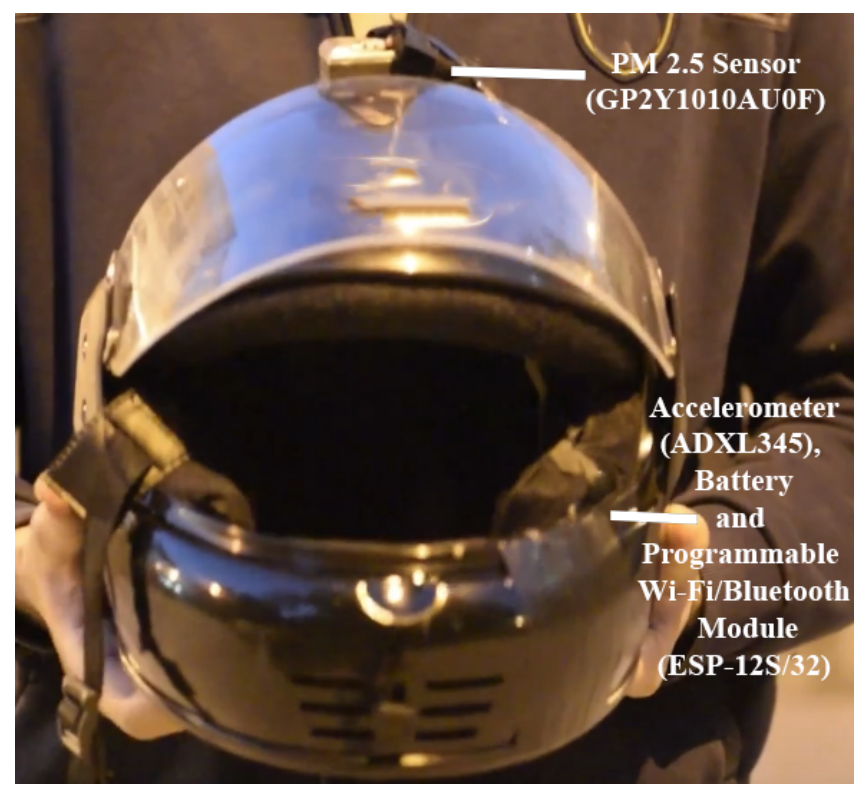

Figure 7. The helmet in its final prototype state. A part of this image is deliberately blurred to avoid intellectual property rights violation.

possible way to forgo this data would be a manual deletion from the database. Furthermore false alarming could be caused by an user (due to curiosity or mischievous behavior) leading to wastage of time and resource of the concerned road authority who could have utilized these on filling up an actual pothole. We acknowledge the problem of stale/invalid pothole 
data, and to avoid this problem, we propose the concept of remembrance factor and severity index as a data validation feature which could be integrated any pothole detection systems as well.

Whenever a SmartPPM user reports a pothole, we set the pothole's remembrance factor $\left(R_{f}\right)$ to 1 ; on the subsequent days, we reduce the value of $R_{f}$ following a one-sided raised cosine defined in (1).

$$
R_{f}(d)= \begin{cases}1, & 0<d \leq \frac{1-\beta}{2 T} \\ \frac{1}{2}\left[1+\cos \left(\frac{\pi T}{\beta}\left[d-\frac{1-\beta}{2 T}\right]\right)\right], & \frac{1-\beta}{2 T}<d \leq \frac{1+\beta}{2 T} \\ 0, & \text { otherwise }\end{cases}
$$

where $R_{f}$ is a function of elapsed time $d$ (days), $T$ $\left(\right.$ days $\left.^{-1}\right)$ which is equal to the inverse of the time after which the reported pothole data become invalid, $\beta$ (roll-off factor) which could be set using the average discovery/action time $d_{\text {act }}$ (days) for the authorities of that area to respond about a reported pothole as

$$
\beta=1-2 T d_{\text {act }} \text {. }
$$

It could be easily shown that $\beta$ will be a smaller value if on average, the authorities are slow to respond about the report of a pothole as $T$ should be set high if the average time taken by the authorities to fill up the potholes is high. The invalid data after $T$ days could be conditionally set for automatic deletion to flush out stale data from the database, which would otherwise remain forever once reported. The factor $T$ and $d_{\text {act }}$ also make the approach of handling stale data highly adaptive as they could be set independently based on pothole's geographic locality. Fig. 8 represents several examples of the distribution of $R_{f}$ with respect to elapsed time $d \in\left[0, \frac{1}{T}\right]$ days which is visualized with different values of $\beta$ and $T=\frac{1}{20}$ days $^{-1}$.

Particularly to deal with the invalid pothole data we have introduced severity index, which is the sum of $R_{f}$ of all independently reported potholes within a radius of $15 \mathrm{~m}$ (which is also the usual uncertainty of the reported uncompensated GPS location [27]) assuming the central coordinate be the oldest reported pothole in the direction of approach. For a false alarm the severity index is expected to be very low, as in the absence of a trigger (i.e., a pothole) the probability of multiple reports of pothole at a particular location would be very low. The user will be notified through patterned mobile phone vibrations, if severity index comes to be greater than a threshold. This threshold could be efficiently learned when more people gets into the system (in our case we have set this threshold be 5 as the number of user were limited during our prototype testing). The severity index could also be utilized by road authorities to prioritize the filling of a pothole based on its value, i.e., higher the severity index more priority should be

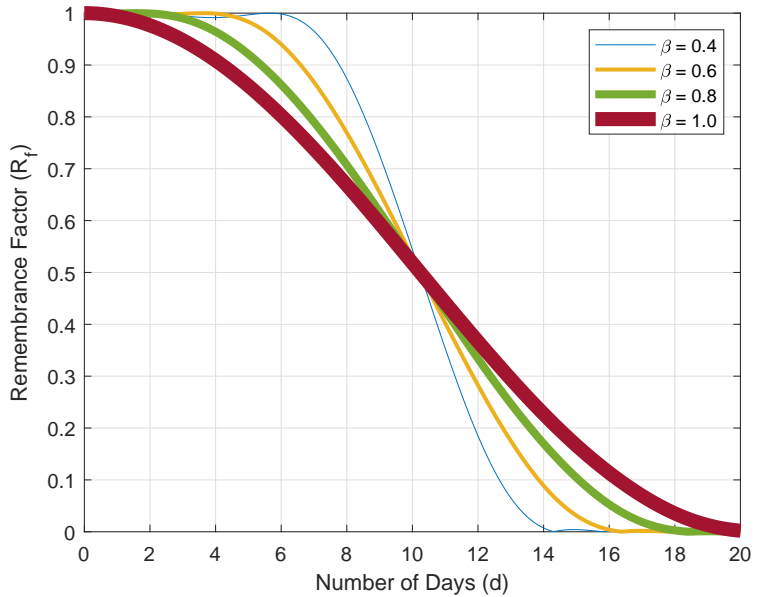

Figure 8. The value of remembrance factor $R_{f}$ as a function of elapsed days $d, T=\frac{1}{20}$ days $^{-1}$ and with different values of $\beta$.

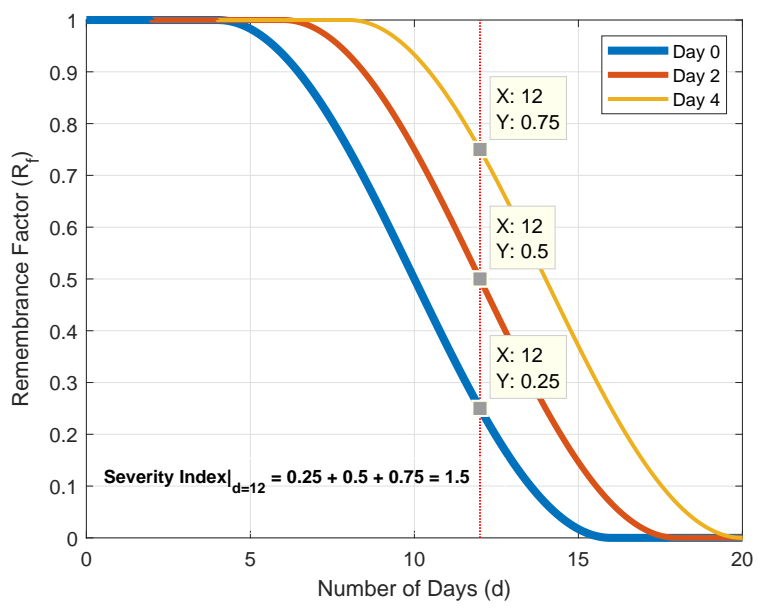

Figure 9. An example calculation of the severity index with three independent reported potholes of a particular location at $0 t h$, $2 n d$, and $4 t h$ day respectively, by setting $d_{a c t}=4$ days, and $T=\frac{1}{20}$ day $^{-1}$ at $d=12$ day.

given to fill the pothole. As an example, to calculate the severity index with three independent reported potholes of a particular location at $0 t h, 2 n d$, and $4 t h$ day respectively, we set $d_{\text {act }}=4$ days, and $T=\frac{1}{20}$ day $^{-1}$ to obtain $\beta=0.6$. At $d=12$ day from the report of the first pothole we obtain,

$$
\begin{aligned}
\text { severity index }\left.\right|_{d=12} & =R_{f}(d)+R_{f}(d-2)+R_{f}(d-4) \\
& =0.25+0.50+0.75=1.50,
\end{aligned}
$$

which is also shown in Fig. 9. As the database resides in the cloud, all the processing involved in updating $R_{f}$ and severity index could be done in the cloud itself. With all the mentioned features, our prototype SmartPPM can improve the on-road safety from potholes and 
reduce the on-road pollution-related health hazards in the real world.

\section{Result Analysis}

Fig. 6.(a) shows the bar chart representing the monthly number of discovered potholes by a SmartPPM user in our trial runs of the prototype from June to December 2018. This bar chart act as a personal performance indicator for a user. The reported pothole data could also be concurrently viewed in terms of geographical location pins over a map.

Fig. 6.(b), shows the average on-road air pollution (AQI) encountered by a SmartPPM's user during the trial runs on the roads of New Delhi (India). Through this screen, a user can visualize the average on-road pollution level he has to encounter in a month and how many times the 250 AQI level is crossed, which is a good indicator of a possible route change for the daily commute.

As our SmartPPM system doesn't automatically detect the pothole but rather through a manual feedback (head-nodding pattern), the accuracy depend on an individual user's performance. In our trial run we noticed that different users perceive potholes differently, as some are hypersensitive to report every possible pothole encounter on the road and some will only report if the encountered pothole is really severe.

\section{Conclusion}

Our prototype serves multiple purposes: monitoring of potholes and real-time location-based air pollution monitoring along-with on-time alert of existing potholes. The proposed prototype is also an economical solution, as it requires a few components to build up, and the compact form factor allow them to be embedded in a helmet very conveniently. The mobile application developed for this system provides timely alerts while approaching a dangerous pothole and reports to the user about the average air pollution the rider has to face while on the go. The data of the pothole and pollution provided by the system could also be utilized by various satellite navigation routing applications to ensure a safer and relatively less polluted route. At last, we have also proposed a very novel remembrance factor and severity index, which could effectively handle stale/invalid pothole data in the system's database.

Acknowledgement. Authors are thankful to Agam, Siddhant, and technical team of CircuitUncle.com for their valuable contributions in this project.

\section{References}

[1] Ministry of Road Transport and Highways (2019) Basic Road Statistics of India (2016-17). Report, Government of India [Online] (accessed on 15 March 2020).
[2] Ministry of Road Transport and Highways (2019) Annual Report 2018-19. Report, Government of India [Online] (accessed on 15 March 2020).

[3] Ministry of Road Transport and Highways (2019) Road Accidents in India - 2018. Report, Government of India [Online] (accessed on 15 March 2020).

[4] Dash, D.K. (2018) Potholes killed 3,597 across India in 2017, terror 803. The Times of India [Online] (accessed on 15 March 2020).

[5] Burningham, S. and Stankevich, N. (2005) Why road maintenance is important and how to get it done. World Bank Transport Notes (The World Bank) TRN-4: 1-10.

[6] Dong, Q., Onyango, M. A. and Huang, B. (2013) Investigation on Service Time and Effective Cost of Typical Pothole Patches in Tennessee. In Proceedings of the International Symposium of Climatic Effects on Pavement and Geotechnical Infrastructure (ASCE): 1-7.

[7] Bhatia, Y., Rai, R., Gupta, V., Aggarwal, N. and Akula, A. (2019) Convolutional neural networks based potholes detection using thermal imaging. Journal of King Saud University-Computer and Information Sciences (Elsevier) 2019(02): 1-11.

[8] Koch, C., Georgieva, K., Kasireddy, V., Akinci, B. and Fieguth, P. (2015) A review on computer vision based defect detection and condition assessment of concrete and asphalt civil infrastructure. Advanced Engineering Informatics (Elsevier) 29(2): 196-210.

[9] Azhar, K., Murtaza, F., Yousaf, M.H. and Habib, H.A. (2016) Computer vision based detection and localization of potholes in asphalt pavement images. In Proceedings of the IEEE Canadian Conference on Electrical and Computer Engineering (CCECE) (IEEE): 1-5.

[10] Christian, K. and Brilakis, I. (2011) Pothole detection in asphalt pavement images. Advanced Engineering Informatics (Elsevier) 25(3): 507-515.

[11] Hoang, N.D., (2018) An artificial intelligence method for asphalt pavement pothole detection using least squares support vector machine and neural network with steerable filter-based feature extraction. Advances in Civil Engineering (Hindawi) 2018: 1-12.

[12] Maeda, H., Sekimoto, Y., Seto, T., Kashiyama, T. and Omata, H. (2018) Road Damage Detection and Classification Using Deep Neural Networks with Smartphone Images. Computer-Aided Civil and Infrastructure Engineering (Wiley) 33(12): 1127-1141.

[13] Hadjidemetriou, G.M., Vela, P.A.and Christodoulou, S.E. (2018) Automated Pavement Patch Detection and Quantification Using Support Vector Machines. Journal of Computing in Civil Engineering (ASCE) 32(1): 1-5.

[14] Ren, J. and Liu, D. (2016) PADS: a reliable pothole detection system using machine learning. In Proceedings of the International Conference on Smart Computing and Communication (Springer): 327-338.

[15] Das, P.D. and Sengupta, S. (2016) Proposing the systems to provide protection of vehicles against theft and accident. In Proceedings of the IEEE International Conference on Recent Trends in Electronics, Information and Communication Technology (RTEICT) (IEEE): 16811685.

[16] Batista, G.E.A.P.A, Ronaldo, C.P. and Monard, M.C. (2004) A study of the behavior of several methods for 
balancing machine learning training data. ACM SIGKDD explorations newsletter (ACM) 6(1): 20-29.

[17] Mednis, A., Strazdins, G., Zviedris, R., Kanonirs, G. and Selavo, L. (2011) Real time pothole detection using Android smartphones with accelerometers. In Proceedings of the International Conference on Distributed Computing in Sensor Systems and Workshops (DCOSS) (IEEE): 1-6.

[18] Madli, R., Hebbar, S., Pattar, P. and Golla, V. (2015) Automatic Detection and Notification of Potholes and Humps on Roads to Aid Drivers. IEEE Sensors Journal (IEEE) 15(8): 4313-4318.

[19] Strutu, M., Stamatescu, G. and Popescu, D. (2013) A mobile sensor network based road surface monitoring system. In Proceedings of the International Conference on System Theory, Control and Computing (ICSTCC) (IEEE): 630-634.

[20] UN Enable (2020) Sustainable Development GoalsUnited Nations. Report, Envision 2030 United Nations [Online] (accessed on 15 March 2020).

[21] Rainio, J., Sulander, P., Hantula, L., Nuutinen, J. and Karkola, K. (2007) Diseases and motor vehicle fatalities in Finland in 2001 and 2002. Traffic Injury Prevention
(T\&F) 8(3): 321-328

[22] Ai-Thinker Technology (2018) Datasheet ESP-12S. Datasheet [0nline] (accessed on 15 March 2020).

[23] Mesquita, J., Guimarães, D., Pereira, P., Santos, F. and Almeida, L. (2018) Assessing the ESP8266 WiFi module for the Internet of Things. In Proceedings of the IEEE 23rd International Conference on Emerging Technologies and Factory Automation (ETFA) (IEEE): 784-791.

[24] Maier, A., Sharp, A. and Vagapov, Y. (2017) Comparative analysis and practical implementation of the ESP32 microcontroller module for the internet of things. In Proceedings of the Internet Technologies and Applications (ITA) (IEEE): 143-148.

[25] Analog Devices (2010) ADXL345 datasheet. Datasheet, [0nline] (accessed on 15 March 2020).

[26] SHARP Corporation (2006) Compact Optical Dust Sensor GP2Y1010AU0F. Datasheet [0nl ine] (accessed on 15 March 2020).

[27] Ross, R. and Hoque, R. (2020) Augmenting GPS with Geolocated Fiducials to Improve Accuracy for Mobile Robot Applications. Applied Sciences (MDPI) 10(1): 1-13. 